\title{
Regarding the axial-vector mesons
}

\author{
De-Min $\mathrm{Li}^{1,2 *}$ Bing $\mathrm{Ma}^{1}$, Hong $\mathrm{Yu}^{2}$ \\ ${ }^{1}$ Department of Physics, Zhengzhou University, Zhengzhou, Henan 450052, P. R. China ${ }^{\dagger}$ \\ ${ }^{2}$ Institute of High Energy Physics, Chinese Academy of Sciences, Beijing 100039, P. R. China
}

July 28, 2021

\begin{abstract}
The implications of the $f_{1}(1285)-f_{1}(1420)$ mixing for the $K_{1}\left({ }^{3} P_{1}\right)-K_{1}\left({ }^{1} P_{1}\right)$ mixing angle is investigated. Based on the $f_{1}(1285)-f_{1}(1420)$ mixing angle $\sim 50^{\circ}$ suggested from the analysis for a substantial body of data concerning the $f_{1}(1420)$ and $f_{1}(1285)$, the masses of the $K_{1}\left({ }^{3} P_{1}\right)$ and $K_{1}\left({ }^{1} P_{1}\right)$ are determined to be $\sim 1307.35 \pm 0.63 \mathrm{MeV}$ and $1370.03 \pm 9.69$ $\mathrm{MeV}$, respectively, which therefore suggests that the $K_{1}\left({ }^{3} P_{1}\right)-K_{1}\left({ }^{1} P_{1}\right)$ mixing angle is about $\pm(59.55 \pm 2.81)^{\circ}$. Also, it is found that the mass of the $h_{1}^{\prime}\left({ }^{1} P_{1}\right)$ (mostly of $s \bar{s}$ ) state is about $1495.18 \pm 8.82 \mathrm{MeV}$. Comparison of the predicted results and the available experimental information of the $h_{1}(1380)$ shows that without further confirmation on the $h_{1}(1380)$, the assignment of the $h_{1}(1380)$ as the $s \bar{s}$ member of the ${ }^{1} P_{1}$ meson nonet may be premature.
\end{abstract}

Key words: axial-vector mesons; hadron mass

PACS numbers:14.40.Ev; 12.40.Yx

*E-mail: lidm@zzu.edu.cn

${ }^{\dagger}$ Mailing address 


\section{Introduction}

The strange axial vector mesons provide interesting possibilities to study the QCD in the nonperturbative regime by the mixing of the ${ }^{3} P_{1}$ and ${ }^{1} P_{1}$ states. In the exact $\mathrm{SU}(3)$ limit, the $K_{1}\left({ }^{3} P_{1}\right)$ and $K_{1}\left({ }^{1} P_{1}\right)$ do not mix, just as the $a_{1}$ and $b_{1}$ mesons do not mix. For the strange quark mass greater than the up and down quark masses so that $\mathrm{SU}(3)$ is broken, also, the $K_{1}\left({ }^{3} P_{1}\right)$ and $K_{1}\left({ }^{1} P_{1}\right)$ do not possess definite C-parity, therefore these states can in principle mix to give the physical $K_{1}(1270)$ and $K_{1}(1400)$.

In the literature, the mixing angle of the $K_{1}\left({ }^{3} P_{1}\right)$ and $K_{1}\left({ }^{1} P_{1}\right), \theta_{K}$ has been estimated by some different approaches, however, there is not yet a consensus on the value of $\theta_{K}$. As the optimum fit to the data as of 1977 , Carnegie et al. finds $\theta_{K}=(41 \pm 4)^{\circ}[1]$. Within the heavy quark effective theory Isgur and Wise predict two possible mixing angles, $\theta_{K} \sim 35.3^{\circ}$ and $\theta_{K} \sim-54.7^{\circ}[2]$. Based on the analysis of $\left.\tau \rightarrow \nu K_{1}(1270)\right)$ and $\left.\tau \rightarrow \nu K_{1}(1400)\right)$, Rosner suggests $\theta_{K} \sim 62^{\circ}[3]$, Asner et al. gives $\theta_{K}=(69 \pm 16 \pm 19)^{\circ}$ or $(49 \pm 16 \pm 19)^{\circ}[4]$, and Cheng obtains $\theta_{K}= \pm 37^{\circ}$ or $\pm 58^{\circ}[5]$. From the experimental information on masses and the partial rates of $K_{1}(1270)$ and $K_{1}(1400)$, Suzuki finds two possible solutions with a two-fold ambiguity, $\theta_{K} \sim 33^{\circ}$ or $57^{\circ}[6]$. A constraint $35^{\circ} \leq \theta_{K} \leq 55^{\circ}$ is predicted by Burakovsky et

al. in a nonrelativistic constituent quark model[7], and within the same model, the values of $\theta_{K} \simeq(31 \pm 4)^{\circ}$ and $\theta_{K} \simeq(37.3 \pm 3.2)^{\circ}$ are suggested by Chliapnikov[8] and Burakovsky[9], respectively. The calculations for the strong decays of $K_{1}(1270)$ and $K_{1}(1400)$ in the ${ }^{3} P_{0}$ decay model suggests $\theta_{K} \sim 45^{\circ}[10,11]$. The mixing angles $\theta_{K} \sim 34^{\circ}[12], \theta_{K} \sim 5^{\circ}[13]$ are also presented within a relativized quark model. More recently, Vijande et al. suggests $\theta_{K} \sim 55.7^{\circ}$ based on the calculations in a constituent quark model[14].

It is widely believed that the $f_{1}(1285)$ and $f_{1}(1420)$ are the isoscalar states of the ${ }^{3} P_{1}$ meson nonet[15]. The analysis of the Gell-Mann-Okubo mass formula, SU(3) coupling formula, radiative decay of the $f_{1}(1285), \gamma \gamma^{*}$ decays of the $f_{1}(1285)$ and $f_{1}(1420)$, and the radiative $J / \psi$ decays performed by Close and Kirk[16] indicates that these various data are independently consistent with the $f_{1}(1285)-f_{1}(1420)$ mixing angle $\alpha \sim 50^{\circ}$ (in the singlet-octet basis). This value of $\alpha \sim 50^{\circ}$ is also supported by the calculations performed by [14, 17, 18, 19]. 
We shall show below that the mass of the $K_{1}\left({ }^{3} P_{1}\right)$ can be related to the mass matrix describing the mixing of the $f_{1}(1285)$ and $f_{1}(1420)$, and the $f_{1}(1285)-f_{1}(1420)$ mixing angle can give a constraint on the mixing of $K_{1}\left({ }^{3} P_{1}\right)-K_{1}\left({ }^{1} P_{1}\right)$. The main purpose of the present work is to discuss the implications of the $f_{1}(1285)-f_{1}(1420)$ mixing for the $K_{1}\left({ }^{3} P_{1}\right)-K_{1}\left({ }^{1} P_{1}\right)$ mixing angle.

\section{The mixing angle of the $K_{1}\left({ }^{3} P_{1}\right)$ and $K_{1}\left({ }^{1} P_{1}\right)$}

In the $N=(u \bar{u}+d \bar{d}) / \sqrt{2}, S=s \bar{s}$ basis, the mass-squared matrix describing the mixing of the $f_{1}(1420)$ and $f_{1}(1285)$ can be written as[20]

$$
M^{2}=\left(\begin{array}{cc}
M_{a_{1}\left({ }^{3} P_{1}\right)}^{2}+2 \beta & \sqrt{2} \beta X \\
\sqrt{2} \beta X & 2 M_{K_{1}\left({ }^{3} P_{1}\right)}^{2}-M_{a_{1}\left({ }^{3} P_{1}\right)}^{2}+\beta X^{2}
\end{array}\right),
$$

where $M_{a_{1}\left({ }^{3} P_{1}\right)}$ and $M_{K_{1}\left({ }^{3} P_{1}\right)}$ are the masses of the states $a_{1}\left({ }^{3} P_{1}\right)$ and $K_{1}\left({ }^{3} P_{1}\right)$, respectively; $\beta$ denotes the total annihilation strength of the $q \bar{q}$ pair for the light flavors $u$ and $d ; X$ describes the $\mathrm{SU}(3)$-breaking ratio of the nonstrange and strange quark propagators via the constituent quark mass ratio $m_{u} / m_{s}$. The masses of the two physical isoscalar states $f_{1}(1420)$ and $f_{1}(1285)$, $M_{1}$ and $M_{2}$, can be related to the matrix $M^{2}$ by the unitary matrix $U$

$$
M^{2}=U^{\dagger}\left(\begin{array}{cc}
M_{1}^{2} & 0 \\
0 & M_{2}^{2}
\end{array}\right) U
$$

and the physical states $f_{1}(1420)$ and $f_{1}(1285)$ can be expressed as

$$
\left(\begin{array}{c}
f_{1}(1420) \\
f_{1}(1285)
\end{array}\right)=U\left(\begin{array}{c}
N \\
S
\end{array}\right)
$$

Also, in the basis $\mathbf{8}=(u \bar{u}+d \bar{d}-2 s \bar{s}) / \sqrt{6}, \mathbf{1}=(u \bar{u}+d \bar{d}+s \bar{s}) / \sqrt{3}$, the mixing of the $f_{1}(1420)$ and $f_{1}(1285)$ can expressed by

$$
\left(\begin{array}{c}
f_{1}(1420) \\
f_{1}(1285)
\end{array}\right)=\left(\begin{array}{cc}
\cos \alpha & -\sin \alpha \\
\sin \alpha & \cos \alpha
\end{array}\right)\left(\begin{array}{l}
\mathbf{8} \\
\mathbf{1}
\end{array}\right)
$$

where $\alpha$ is the $f_{1}(420)-f_{1}(1285)$ mixing angle in the singlet-octet basis.

With the help of

$$
\left(\begin{array}{l}
\mathbf{8} \\
\mathbf{1}
\end{array}\right)=\left(\begin{array}{cc}
\sqrt{\frac{1}{3}} & -\sqrt{\frac{2}{3}} \\
\sqrt{\frac{2}{3}} & \sqrt{\frac{1}{3}}
\end{array}\right)\left(\begin{array}{l}
N \\
S
\end{array}\right)
$$


from (3) and (4), one can have

$$
U=\left(\begin{array}{cc}
\cos \alpha & -\sin \alpha \\
\sin \alpha & \cos \alpha
\end{array}\right)\left(\begin{array}{cc}
\sqrt{\frac{1}{3}} & -\sqrt{\frac{2}{3}} \\
\sqrt{\frac{2}{3}} & \sqrt{\frac{1}{3}}
\end{array}\right) .
$$

Based on (1), (2) and (6), the following relations can be obtained

$$
\begin{gathered}
M_{a_{1}\left({ }^{3} P_{1}\right)}^{2}+2 \beta=\left(\sqrt{\frac{1}{3}} \cos \alpha-\sqrt{\frac{2}{3}} \sin \alpha\right)^{2} M_{1}^{2}+\left(\sqrt{\frac{2}{3}} \cos \alpha+\sqrt{\frac{1}{3}} \sin \alpha\right)^{2} M_{2}^{2}, \\
\sqrt{2} \beta X=\left(\sqrt{\frac{1}{3}} \cos \alpha-\sqrt{\frac{2}{3}} \sin \alpha\right)\left(\sqrt{\frac{2}{3}} \cos \alpha+\sqrt{\frac{1}{3}} \sin \alpha\right)\left(M_{2}^{2}-M_{1}^{2}\right), \\
2 M_{K_{1}\left({ }^{3} P_{1}\right)}^{2}-M_{a_{1}\left({ }^{3} P_{1}\right)}^{2}+\beta X^{2}=\left(\sqrt{\frac{1}{3}} \cos \alpha-\sqrt{\frac{2}{3}} \sin \alpha\right)^{2} M_{2}^{2}+\left(\sqrt{\frac{2}{3}} \cos \alpha+\sqrt{\frac{1}{3}} \sin \alpha\right)^{2} M_{1}^{2} .
\end{gathered}
$$

The constituent quark mass ratio can be determined within the nonrelativistic constituent quark model(NRCQM). In NRCQM[8, 9], the mass of a $q \bar{q}$ state with $L=0, M_{q \bar{q}}$ is given by

$$
M_{q \bar{q}}=m_{q}+m_{\bar{q}}+\Lambda \frac{\mathbf{s}_{q} \cdot \mathbf{s}_{\bar{q}}}{m_{q} m_{\bar{q}}}
$$

where $m$ and $\mathbf{s}$ are the constituent quark mass and spin, $\Lambda$ is a constant. Since $\mathbf{s}_{q} \cdot \mathbf{s}_{\bar{q}}=-3 / 4$ for spin- 0 mesons and $1 / 4$ for spin- 1 mesons, in the $\mathrm{SU}(2)$ flavor symmetry limit, one can have

$$
X \equiv \frac{m_{u}}{m_{s}}=\frac{M_{\pi}+3 M_{\rho}}{2 M_{K}+6 M_{K^{*}}-M_{\pi}-3 M_{\rho}}=0.6298 \pm 0.00068 .
$$

Taking $\alpha \simeq 50^{\circ}$ obtained from several independent analyses[16] as mentioned in section 1 , $M_{1}=1426.3 \pm 0.9 \mathrm{MeV}$ and $M_{2}=1281.8 \pm 0.6 \mathrm{MeV}[15]$, from relations (7)-(9), we have ${ }^{1}$

$$
M_{K_{1}\left({ }^{3} P_{1}\right)} \simeq 1307.35 \pm 0.63 \mathrm{MeV}, \quad M_{a_{1}\left({ }^{3} P_{1}\right)} \simeq 1205.06 \pm 0.92 \mathrm{MeV}
$$

The $K_{1}\left({ }^{3} P_{1}\right)$ and $K_{1}\left({ }^{1} P_{1}\right)$ can mix to produce the physical states $K_{1}(1400)$ and $K_{1}(1270)$ and the mixing between $K_{1}\left({ }^{3} P_{1}\right)$ and $K_{1}\left({ }^{1} P_{1}\right)$ can be parameterized as $[6]$

$$
\begin{aligned}
& K_{1}(1400)=K_{1}\left({ }^{3} P_{1}\right) \cos \theta_{K}-K_{1}\left({ }^{1} P_{1}\right) \sin \theta_{K}, \\
& K_{1}(1270)=K_{1}\left({ }^{3} P_{1}\right) \sin \theta_{K}+K_{1}\left({ }^{1} P_{1}\right) \cos \theta_{K},
\end{aligned}
$$

where $\theta_{K}$ denotes the $K_{1}\left({ }^{3} P_{1}\right)-K_{1}\left({ }^{1} P_{1}\right)$ mixing angle. Without any assumption about the origin of the $K_{1}\left({ }^{3} P_{1}\right)-K_{1}\left({ }^{1} P_{1}\right)$ mixing, the masses of the $K_{1}\left({ }^{3} P_{1}\right)$ and $K_{1}\left({ }^{1} P_{1}\right)$ can be related

\footnotetext{
${ }^{1}$ Here $\beta \simeq 108078.0 \pm 834.788 \mathrm{MeV}^{2}$.
} 
to $M_{K_{1}(1400)}$ and $M_{K_{1}(1270)}$, the masses of the $K_{1}(1400)$ and $K_{1}(1270)$, by the following relation phenomenologically,

$$
S\left(\begin{array}{cc}
M_{K_{1}\left({ }^{3} P_{1}\right)}^{2} & A \\
A & M_{K_{1}\left({ }^{1} P_{1}\right)}^{2}
\end{array}\right) S^{\dagger}=\left(\begin{array}{cc}
M_{K_{1}(1400)}^{2} & 0 \\
0 & M_{K_{1}(1270)}^{2}
\end{array}\right)
$$

where $A$ denotes a parameter describing the $K_{1}\left({ }^{3} P_{1}\right)-K_{1}\left({ }^{1} P_{1}\right)$ mixing , and

$$
S=\left(\begin{array}{cc}
\cos \theta_{K} & -\sin \theta_{K} \\
\sin \theta_{K} & \cos \theta_{K}
\end{array}\right)
$$

From (14), one can have

$$
\begin{aligned}
& M_{K_{1}\left({ }^{3} P_{1}\right)}^{2}=M_{K_{1}(1400)}^{2} \cos ^{2} \theta_{K}+M_{K_{1}(1270)}^{2} \sin ^{2} \theta_{K}, \\
& M_{K_{1}\left({ }^{1} P_{1}\right)}^{2}=M_{K_{1}(1400)}^{2} \sin ^{2} \theta_{K}+M_{K_{1}(1270)}^{2} \cos ^{2} \theta_{K}, \\
& \cos \left(2 \theta_{K}\right)=\frac{M_{K_{1}\left({ }^{3} P_{1}\right)}^{2}-M_{K_{1}\left({ }^{1} P_{1}\right)}^{2}}{M_{K_{1}(1400)}^{2}-M_{K_{1}(1270)}^{2}} .
\end{aligned}
$$

Inputting $M_{K_{1}(1400)}=1402 \pm 7 \mathrm{MeV}, M_{K_{1}(1270)}=1273 \pm 7 \mathrm{MeV}[15]$ and $M_{K_{1}\left({ }^{3} P_{1}\right)} \simeq 1307.35 \pm$ $0.63 \mathrm{MeV}$ shown in (12), from (15)-(17), we have

$$
M_{K_{1}\left({ }^{1} P_{1}\right)} \simeq 1370.03 \pm 9.69 \mathrm{MeV}, \quad\left|\theta_{K}\right| \simeq(59.55 \pm 2.81)^{\circ} .
$$

Recently, based on the relations (15)-(17) and restricting to $0<\theta_{K}<90^{\circ}$, Nardulli and Pham found $[21]$

$$
\begin{aligned}
& \text { [solution a]: }\left(M_{K_{1}\left({ }^{1} P_{1}\right)}, M_{K_{1}\left({ }^{3} P_{1}\right)}\right)=(1310,1367) \mathrm{MeV}, \text { for } \theta_{K}=32^{\circ}, \\
& \text { [solution b]: }\left(M_{K_{1}\left({ }^{1} P_{1}\right)}, M_{K_{1}\left({ }^{3} P_{1}\right)}\right)=(1367,1310) \mathrm{MeV} \text {, for } \theta_{K}=58^{\circ} .
\end{aligned}
$$

Our predicted result that $\left(M_{K_{1}\left({ }^{1} P_{1}\right)}, M_{K_{1}\left({ }^{3} P_{1}\right)}\right) \simeq(1370,1307) \mathrm{MeV}$ and $\left|\theta_{K}\right| \simeq 59.55^{\circ}$ extracted from $\alpha \simeq 50^{\circ}$ is in excellent agreement with the solution $\mathrm{b}$ given by[21].

Within the nonrelativistic constituent quark model, the results regarding the masses of the $K_{1}\left({ }^{1} P_{1}\right)$ and $K_{1}\left({ }^{3} P_{1}\right),\left(M_{K_{1}\left({ }^{1} P_{1}\right)}, M_{K_{1}\left({ }^{3} P_{1}\right)}\right)=(1368,1306) \mathrm{MeV}$ suggested by [8] and $\left(M_{K_{1}\left({ }^{1} P_{1}\right)}, M_{K_{1}\left({ }^{3} P_{1}\right)}\right)=(1356,1322) \mathrm{MeV}$ suggested by [9], are in good agreement with our predicted result. However, based on the following relation employed by [8, 9]

$$
\tan ^{2}\left(2 \theta_{K}\right)=\left(\frac{M_{K_{1}\left({ }^{3} P_{1}\right)}^{2}-M_{K_{1}\left({ }^{1} P_{1}\right)}^{2}}{M_{K_{1}(1400)}^{2}-M_{K_{1}(1270)}^{2}}\right)^{2}-1,
$$


the values of $\theta_{K}=(31 \pm 4)^{\circ}$ given by[8] and $\theta_{K}=(37.3 \pm 3.2)^{\circ}$ given by[9] disagree with value of $\left|\theta_{K}\right| \simeq(59.55 \pm 2.81)^{\circ}$ given by the present work.

Obviously, (19) is equivalent to (17), and will yield two solutions $\left|\theta_{K}\right|$ and $\frac{\pi}{2}-\left|\theta_{K}\right|$. Simultaneously considering the relations (15), (16) and (19), in the presence of $M_{K_{1}(1400)}>M_{K_{1}(1270)}$, we can conclude that if $M_{K_{1}\left({ }^{3} P_{1}\right)}<M_{K_{1}\left({ }^{1} P_{1}\right)}$, the $\left|\theta_{K}\right|$ would greater than $45^{\circ}$. In fact, relation (17) clearly indicates that in the presence of $M_{K_{1}(1400)}>M_{K_{1}(1270)}$, the case $M_{K_{1}\left({ }^{3} P_{1}\right)}<M_{K_{1}\left({ }^{1} P_{1}\right)}$ must require $45^{\circ}<\left|\theta_{K}\right|<90^{\circ}$.

In the framework of a covariant light-front quark model, the calculations performed by Cheng and Chua [22] for the exclusive radiative $B$ decays, $B \rightarrow K_{1}(1270) \gamma, K_{1}(1400) \gamma$, show that the relative strength of $B \rightarrow K_{1}(1270) \gamma$ and $B \rightarrow K_{1}(1270) \gamma$ rates is very sensitive to the sign of the $K_{1}(1270)-K_{1}(1400)$ mixing angle. For $\theta_{K}= \pm 58^{\circ}$, the following relation is predicted[22]

$$
\frac{\mathcal{B}\left(B \rightarrow K_{1}(1270) \gamma\right)}{\mathcal{B}\left(B \rightarrow K_{1}(1270) \gamma\right)}= \begin{cases}10.1 \pm 6.2 & \text { for } \theta_{K}=+58^{\circ} \\ 0.02 \pm 0.02 & \text { for } \theta_{K}=-58^{\circ}\end{cases}
$$

Evidently, experimental measurement of the above ratio of branching fractions can be used to fix the sign of the $K_{1}\left({ }^{3} P_{1}\right)-K_{1}\left({ }^{1} P_{1}\right)$ mixing angle. Recently, the first measurement of the branching ratio $\mathcal{B}$ for $B$ decay into $K_{1}(1270) \gamma$, together with an upper bound on $K_{1}(1400), \mathcal{B}\left(B^{+} \rightarrow\right.$ $\left.K_{1}^{+}(1270) \gamma\right)=(4.28 \pm 0.94 \pm 0.43) \times 10^{-5}, \mathcal{B}\left(B^{+} \rightarrow K_{1}^{+}(1400) \gamma\right)<1.44 \times 10^{-5}$ is reported by Belle collaboration[23]. Based on the measurements of Bell collaboration[23], the analysis of the radiative $B$ decays with an axial-vector meson in the final state performed by Nardulli and Pham[21] within naive factorization suggests that $\mathcal{B}\left(B^{+} \rightarrow K_{1}^{+}(1400) \gamma\right)=4.4 \times 10^{-6}$ for $\theta_{K}=+58^{\circ}$, which is consistent with the predictions given by[22]. Further experimental studies of $\mathcal{B}\left(B^{+} \rightarrow K_{1}^{+}(1270) \gamma\right)$ and $\mathcal{B}\left(B^{+} \rightarrow K_{1}^{+}(1400) \gamma\right)$ is certainly desirable for understanding the sign of the $K_{1}\left({ }^{3} P_{1}\right)-K_{1}\left({ }^{1} P_{1}\right)$ mixing angle.

Our predicted center value of the $a_{1}\left({ }^{3} P_{1}\right)$ mass is $\sim 1205.06 \mathrm{MeV}$, slightly smaller than the measured center value of the $a_{1}(1260)$ mass, $1230 \mathrm{MeV}$, although the predicted value $1205.06 \pm 0.92 \mathrm{MeV}$ is consistent with the experimental datum $1230 \pm 40 \mathrm{MeV}$ within errors. The similar result has been obtained by Chliapnikov within NRCQM[8]. According to the NRCQM prediction that if $M_{K_{1}\left({ }^{3} P_{1}\right)}<M_{K_{1}\left({ }^{1} P_{1}\right)}, M_{a_{1}\left({ }^{3} P_{1}\right)}$ would be less than $M_{b_{1}\left({ }^{1} P_{1}\right)}[8,9]$, therefore, in the presence of $M_{K_{1}\left({ }^{3} P_{1}\right)} \simeq 1307<M_{K_{1}\left({ }^{1} P_{1}\right)} \simeq 1370 \mathrm{MeV}$, the $a_{1}\left({ }^{3} P_{1}\right)$ mass should smaller 
than the $b_{1}(1230)$ mass $(1229.5 \pm 3.2 \mathrm{MeV}[15])$. In addition, notice that the determination of the $a_{1}(1260)$ mass in hadronic production and in $\tau \rightarrow a_{1} \nu_{\tau}$ decay is to a certain extent model dependent[15].

\section{The $s \bar{s}$ member of the ${ }^{1} P_{1}$ meson nonet}

According to PDG[15], the $h_{1}(1170)$ as the ${ }^{1} P_{1}$ isoscalar state (mostly of $\left.u \bar{u}+d \bar{d}\right)$ is well established experimentally. However, the assignment of $s \bar{s}$ partner of the $h_{1}(1170)$ remains ambiguous. In the presence of the $b_{1}(1235)$ and $h_{1}(1170)$ being the members of the ${ }^{1} P_{1}$ meson nonet, with the help of the $K_{1}\left({ }^{1} P_{1}\right)$ mass obtained in section 2 , we shall estimate the mass of the ${ }^{1} P_{1} s \bar{s}$ state using different approaches.

By applying (1) and (2) to the ${ }^{1} P_{1}$ meson nonet, we can obtain the following relations

$$
\begin{aligned}
& 2 M_{K_{1}\left({ }^{1} P_{1}\right)}^{2}+\left(2+X^{2}\right) \beta_{1}=M_{h_{1}(1170)}^{2}+M_{h_{1}^{\prime}}^{2}, \\
& \left(M_{b_{1}(1235)}^{2}+2 \beta_{1}\right)\left(2 M_{K_{1}\left({ }^{1} P_{1}\right)}^{2}-M_{b_{1}(1235)}^{2}+\beta_{1} X^{2}\right)-2 \beta_{1}^{2} X^{2}=M_{h_{1}(1170)}^{2} M_{h_{1}^{\prime}}^{2},
\end{aligned}
$$

where $h_{1}^{\prime}$ denotes the $s \bar{s}$ partner of the ${ }^{1} P_{1}$ states $h_{1}(1170)$ and $b_{1}(1235)$. Using $M_{K_{1}\left({ }^{1} P_{1}\right)} \simeq$ $1370.03 \pm 9.69 \mathrm{MeV}, X=0.6298 \pm 0.00068$ obtained in section 2, and the measured values $M_{b_{1}(1235)}=1229.5 \pm 3.2 \mathrm{MeV}$ and $M_{h_{1}(1170)}=1170 \pm 20 \mathrm{MeV}[15]$, we have

$$
\beta_{1} \simeq-(69143.5 \pm 22373.6) \mathrm{MeV}^{2}, \quad M_{h_{1}^{\prime}} \simeq 1489.75 \pm 18.08 \mathrm{MeV}
$$

Then from (1) and (2), the quarkonia content of the $h_{1}(1170)$ and $h_{1}^{\prime}(1490)$ can be given by

$$
\left(\begin{array}{l}
h_{1}^{\prime}(1490) \\
h_{1}(1170)
\end{array}\right) \simeq\left(\begin{array}{ll}
0.073 \pm 0.02 & -(0.997 \pm 0.002) \\
0.997 \pm 0.002 & 0.073 \pm 0.02
\end{array}\right)\left(\begin{array}{l}
N \\
S
\end{array}\right)
$$

(22) and (23) indicate that with the $b_{1}(1230), h_{1}(1170)$ and $K_{1}(1370)$ in the ${ }^{1} P_{1}$ meson nonet, another isoscalar state of the ${ }^{1} P_{1}$ meson nonet, $h_{1}^{\prime}$ would have a mass about $1490 \mathrm{MeV}$ and is composed mostly of $s \bar{s}$.

Considering the fact that the $f_{2}^{\prime}(1525)$ is an almost pure $s \bar{s}$ state[20], we obtain the estimated mass of the ${ }^{1} P_{1} s \bar{s}$ state from the following relation given by NRCQM[8]

$$
M_{s \bar{s}\left({ }^{1} P_{1}\right)}=M_{f_{2}^{\prime}(1525)}-\left(M_{a_{2}(1320)}-M_{b_{1}(1235)}\right) X^{2}=1489.78 \pm 5.16 \mathrm{MeV}
$$

which is in excellent agreement with $M_{h_{1}^{\prime}} \simeq 1489.75 \pm 18.08 \mathrm{MeV}$ shown in (22). 
Also, in the framework of the quasi-linear Regge trajectory (see Ref.[19] and references therein), i.e.,

$$
J=\alpha_{i \bar{i}^{\prime}}(0)+\alpha_{i \bar{i}^{\prime}}^{\prime} M_{i \bar{i}^{\prime}}^{2},
$$

where $i\left(\overline{i^{\prime}}\right)$ refers to the quark (antiquark) flavor, $J$ and $M_{i \bar{i}^{\prime}}$ are respectively the spin and mass of the $i \bar{i}^{\prime}$ meson, $\alpha_{i \bar{i}^{\prime}}(0)$ and $\alpha_{i \bar{i}^{\prime}}^{\prime}$ are respectively the intercept and slope of the trajectory on which the $i \overline{i^{\prime}}$ meson lies; For a meson multiplet, the parameters for different flavors can be related by the following relations

(i) additivity of intercepts,

$$
\alpha_{i \bar{i}}(0)+\alpha_{j \bar{j}}(0)=2 \alpha_{j \bar{i}}(0)
$$

(ii) additivity of inverse slopes,

$$
\frac{1}{\alpha_{i \bar{i}}^{\prime}}+\frac{1}{\alpha_{j \bar{j}}^{\prime}}=\frac{2}{\alpha_{j \bar{i}}^{\prime}}
$$

for the ${ }^{1} P_{1} q \bar{q}$ nonet, one can have ${ }^{2}$

$$
M_{s \bar{s}\left({ }^{1} P_{1}\right)}=\left[\frac{2 \alpha_{n \bar{s}}^{\prime} M_{K_{1}\left({ }^{1} P_{1}\right)}^{2}-\alpha_{n \bar{n}}^{\prime} M_{b_{1}(1235)}^{2}}{\alpha_{s \bar{s}}^{\prime}}\right]^{\frac{1}{2}}=1506.01 \pm 18.62 \mathrm{MeV},
$$

which is also consistent with $M_{h_{1}^{\prime}} \simeq 1489.75 \pm 18.08 \mathrm{MeV}$ given in (22).

In the presence of the $b_{1}(1235), h_{1}(1170)$ and $K_{1}\left({ }^{1} P_{1}\right)$ (with a mass about $1370 \mathrm{MeV}$ ) belonging to the ${ }^{1} P_{1}$ meson nonet, the above three different and complementary approaches, i.e., meson-meson mixing, nonrelativistic constituent quark model and Regge phenomenology, consistently suggest that the ninth member of the ${ }^{1} P_{1}$ nonet has a mass about $1495.18 \pm 8.82$ $\mathrm{MeV}$ (averaged value of the above three predicted results) and is mainly strange. Our predicted mass of the ${ }^{1} P_{1} s \bar{s}$ state is in good agreement with the values $1499 \pm 16 \mathrm{MeV}$ suggested by Chliapnikov in a nonrelativistic constituent quark model[8] and $1511 \mathrm{MeV}$ recently found by Vijande et al. in a constituent quark model[14].

Experimentally, the $h_{1}(1380)$ with $J^{P C}=1^{+-}$was claimed to be observed in $K \bar{K} \pi$ system by only two collaborations, LASS collaboration[24] (Mass: $1380 \pm 20 \mathrm{MeV}, \Gamma=80 \pm 30 \mathrm{MeV}$ ) and Crystal Barrel collaboration[25] (Mass: $1440 \pm 60 \mathrm{MeV}, \Gamma=170 \pm 80 \mathrm{MeV}$ ), and the observed decay mode of the $h_{1}(1380)\left(K \bar{K}^{*}\right)$ favors the assignment of the $h_{1}(1380)$ as a $s \bar{s}$ state.

\footnotetext{
${ }^{2}$ Here we take $\alpha_{n \bar{n}}^{\prime}=0.7218, \alpha_{s \bar{s}}^{\prime}=0.6613$ and $\alpha_{n \bar{s}}^{\prime}=0.6902 \mathrm{GeV}^{-2}[19]$.
} 
On the one hand, our predicted mass of the ${ }^{1} P_{1} s \bar{s}$ state, $1495.18 \pm 8.82 \mathrm{MeV}$, is significantly larger than $1380 \pm 20 \mathrm{MeV}$. The prediction given by Godfrey and Isgur in a relativized quark model[12] for the mass of the ${ }^{1} P_{1} s \bar{s}$ state is $1.47 \mathrm{GeV}$, at least $70 \mathrm{MeV}$ higher than the measured result of LASS[24]. Therefore if the measured results of LASS[24] are confirmed, the $h_{1}(1380)$ seems too light to be the ${ }^{1} P_{1} s \bar{s}$ member. The studies on the implications of large $N_{c}$ and chiral symmetry for the mass spectra of meson resonances performed by Cirigliano et al.[26] also disfavor the assignment of the $h_{1}(1380)$ to ${ }^{1} P_{1} s \bar{s}$.

On the other hand, the predicted mass of the ${ }^{1} P_{1} s \bar{s}$ state is consistent with $1440 \pm 60 \mathrm{MeV}$ within errors, and the calculations performed by Barnes et al.[11] for the total width of the ${ }^{1} P_{1}$ $s \bar{s}$ state in the ${ }^{3} P_{0}$ decay model also show that at this mass the assignment of the $h_{1}(1380)$ as the ${ }^{1} P_{1} s \bar{s}$ state appears plausible. So if the measured results of Crystal Barrel[25] are confirmed, the $h_{1}(1380)$ would be a convincing candidate for the $s \bar{s}$ partner of the ${ }^{1} P_{1}$ state $h_{1}(1170)$.

Notice that the uncertainties of these measurements are rather large, and the $h_{1}(1380)$ state still needs further confirmation[15]. Without confirmed experimental information about the $h_{1}(1380)$, the present results indicate that the assignment of the $h_{1}(1380)$ as the ${ }^{1} P_{1} s \bar{s}$ member may be premature.

\section{Concluding remarks}

The studies on the implications of the $f_{1}(1285)-f_{1}(1420)$ mixing for the $K_{1}\left({ }^{3} P_{1}\right)-K_{1}\left({ }^{1} P_{1}\right)$ mixing angle indicate that the $f_{1}(1285)-f_{1}(1420)$ mixing angle $\sim 50^{\circ}$ suggested by Close et al.[16] implies that $\left(M_{K_{1}\left({ }^{3} P_{1}\right)}, M_{K_{1}\left({ }^{1} P_{1}\right)}\right) \simeq(1307,1370) \mathrm{MeV}$, which therefore suggests that the $K_{1}\left({ }^{3} P_{1}\right)-K_{1}\left({ }^{1} P_{1}\right)$ mixing angle $\simeq \pm 59.55^{\circ}$. Experimental measurement of the ratio of $B \rightarrow K_{1}(1270) \gamma$ and $B \rightarrow K_{1}(1270) \gamma$ rates can be used to fix the sign of the $K_{1}\left({ }^{3} P_{1}\right)-K_{1}\left({ }^{1} P_{1}\right)$ mixing angle. Also, with the $b_{1}(1235), h_{1}(1170)$ and $K_{1}\left({ }^{1} P_{1}\right)$ in the ${ }^{1} P_{1}$ meson nonet, three different and complementary approaches, i.e., meson-meson mixing, nonrelativistic constituent quark model and Regge phenomenology, consistently suggest that the ${ }^{1} P_{1} s \bar{s}$ member has a mass about $1495.18 \mathrm{MeV}$. Our predicted mass of the ${ }^{1} P_{1} s \bar{s}$ state is significantly larger than the measured value of the $h_{1}(1380)$ mass reported by LASS[24], while consistent with that 
reported by Crystal Barrel[25], which shows that without further confirmation on the $h_{1}(1380)$, the assignment of the $h_{1}(1380)$ remains open.

Acknowledgments: This work is supported in part by National Natural Science Foundation of China under Contract No. 10205012, Henan Provincial Science Foundation for Outstanding Young Scholar under Contract No. 0412000300, Henan Provincial Natural Science Foundation under Contract No. 0311010800, and Foundation of the Education Department of Henan Province under Contract No. 2003140025.

\section{References}

[1] R. K. Carnegie et al, Phys. Lett. B 68, 289 (1977)

[2] N. Isgur, M. B. Wise, Phys. Lett. B 232, 113 (1989)

[3] J. Rosner, Com. Nucl. Part. Phys. 16, 109 (1986)

[4] D. M. Asner et al., Phys. Rev. D 62, 072006 (2000)

[5] H. Y. Cheng, Phys. Rev. D 67, 094007 (2003)

[6] M. Suzuki, Phys. Rev. D 47, 1252 (1997)

[7] L. Burkovsky, T. Goldman, Phys. Rev. D 56,1368 (1997)

[8] P. V. Chliapnikov, Phys. Lett. B 496, 129 (2000)

[9] L. Burakovsky, T. Goldman, Phys. Rev. D 57, 2879 (1998)

[10] H. G. Blundell, S. Godfrey, B. Phelps, Phys. Rev. D 53, 3712 (1996)

[11] T. Barnes, N. Black, P. R. Page, Phys. Rev. D 68, 054014 (2003)

[12] S. Godfrey, N. Isgur, Phys. Rev. D 32, 189 (1985)

[13] S. Godfrey, R. Kokoski, Phys. Rev. D 43, 1679 (1991)

[14] J. Vijande, F. Fernandez, A. Valcarce, J. Phys. G 31, 481 (2005)

[15] S. Eidelman et al., Phys. Lett. B 592, 1 (2004)

[16] F. E. Close, A. Kirk, Z. Phys. C 76, 469 (1997)

[17] D. M. Li, H. Yu, Q. X. Shen, Chin. Phys. Lett. 17, 558 (2000)

[18] W. S. Carvalho, A. S. de Castro, A. C. B. Antunes, J. Phys. A 35, 7585 (2002) 
[19] D. M. Li et al., Eur. Phys. J. C 37, 323 (2004)

[20] D. M. Li, H. Yu, Q. X. Shen, J. Phys. G 27, 807 (2001)

[21] G. Nardulli, T. N. Pham, hep-ph/0505048

[22] H. Y. Cheng, C. K. Chua, Phys. Rev. D 69, 094007 (2004)

[23] H. Yang et al., Belle Collaboration, Phys. Rev. Lett. 94, 111802 (2005)

[24] D. Aston et al., LASS Collaboration, Phys. Lett. B 201, 573, (1988)

[25] A. Abel et al., Crystal Barrel Collaboration, Phys. Lett. B 415, 280 (1997)

[26] V. Cirigliano, G. Ecker, H. Neufeld, A. Pich, JHEP 0306, 012 (2003) 